\title{
The opportunity for polymorphism and genic variation in social hymenoptera with worker-produced males
}

\author{
Robin E. Owen*
}

Department of Genetics and Human Variation, La Trobe University, Bundoora, Victoria, Australia 3083

In many eusocial Hymenoptera, for example, bumble bees, a proportion, $\psi$ of the males are produced by the workers. The conditions for there to be at least one stable polymorphic equilibrium were found to be, $w_{11} v_{1}-\frac{1}{2} \psi v_{2}\left(w_{11}-w_{12}\right)<$ $\frac{1}{2} w_{12}\left(v_{1}+v_{1}\right)>w_{22} v_{2}-\frac{1}{2} \psi v_{1}\left(w_{22}-w_{12}\right)$; where $w_{11}, w_{12}, w_{22}$ are respectively the fitnesses of the female genotypes $A_{1} A_{1}$, $A_{1} A_{2}, A_{2} A_{2}$ and $v_{1}, v_{2}$ are the fitnesses of the male genotypes $A_{1}$ and $A_{2}$. The equilibrium gene frequencies are given by the root(s) of a cubic polynomial. The conditions given above were shown to be both sufficient and necessary to find all stable equilibria for three regular selection models analysed in detail: (1) Codominance and (2) Dominance with opposite selection in the sexes (3) Overdominance with identical selection in the sexes. The effect of worker-produced males on the probability of a polymorphism and the expected genic variation was investigated for these three models. Compared to other Hymenoptera, species with worker-produced males will have a reduced frequency of polymorphisms and lower mean genic diversity at loci with dominant and overdominant fitnesses. In contrast at loci with codominant fitnesses the probability of a polymorphism and the genic diversity will be higher if the selective differentials are large $(0-1 \cdot 0)$, with smaller selective differentials $(0-0 \cdot 125,0-0 \cdot 01)$ a reduction will again occur. Therefore the overall level of genic diversity depends on the proportion of codominant loci and the selection intensity.

\section{INTRODUCTION}

The insect order Hymenoptera has a haplodiploid genetic system in which (diploid) females are derived from fertilised eggs while the (haploid) males arise by parthenogenesis from unfertilised eggs. Thus it is equivalent to inheritance at $X$ linked loci in diplodiploid species.

Over the last few years it has become apparent that at allozyme loci both solitary and social Hymenoptera have significantly lower levels of mean heterozygosity or genic variation than diplodiploid insects (Snyder, 1974; Metcalf, Marlin and Whitt, 1975; Pamilio, Varvio-Aho and Pekkarinen, 1978; Lester and Selander, 1979; Berkelhamer, 1983). This difference in the level of genic variation is predicted by population genetics theory on a number of grounds, none of which are mutually exclusive.

Haplodiploids have a reduced effective population size $N_{e}$, compared to diplodiploids and so neutral alleles will be lost from the population more rapidly (Mayo, 1976; Crozier, 1979).

* Present address: Department of Biology, University of Calgary, Calgary, Alberta, Canada T2N 1 N4
However the generality of this is doubtful because $N_{e}$ varies greatly with sex ratio and with highly female biased sex ratios haplodiploids have a larger $N_{e}$ than diplodiploids (Crozier, 1976). Lester and Selander (1979) argue that the reduction in $N_{e}$ and lower recombination rate in haplodiploids will lead to a stronger hitchhiking effect and thus a faster elimination of neutral alleles than in diplodiploids.

Deleterious recessive alleles in a state of mutation-selection balance are also predicted to be at a lower frequency in haplodiploids than in diplodiploids because each generation the alleles are exposed to selection in the haploid males (Crozier, 1979). A modified version of Ohta and Kimura's (1975) charge-state model IV (Pamilo and Crozier, 1981) gives a similar result.

It is well known that the conditions for a balanced polymorphism at a diallelic locus undergoing selection are more stringent for $X$-linked or haplodiploid loci than for autosomal loci (Haldane, 1926; Bennett, 1957, 1958; Mandel, 1959). In haplodiploids overdominance is neither necessary nor sufficient for maintaining a stable polymorphic equilibrium (Bennett, 1957). If the 
fitnesses of the corresponding hemizygotes and homozygotes are equal then haplodiploids can maintain fewer polymorphisms by overdominance than diplodiploids (Hartl, 1971). The situation is more complicated for polymorphisms not maintained by overdominance because there is necessarily differential selection between the sexes in diplodiploids (Owen, 1953) as well as in haplodiploids. Pamilo (1979) analysed, in addition to the overdominance model, codominance and dominance models with opposite selection in the sexes for haplodiploid and diplodiploid loci (see table 1). He found that in each model the portion

Table 1 The fitness values of the genotypes in the three regular selection models considered by Lester (1975) and Pamilo (1979)

\begin{tabular}{lllllll}
\hline & \multicolumn{3}{c}{ Females } & & \multicolumn{2}{c}{ Males } \\
\cline { 2 - 3 } Model & $A_{1} A_{1}$ & $A_{1} A_{2}$ & $A_{2} A_{2}$ & & $A_{1}$ & $A_{2}$ \\
\hline Codominance & 1 & $1-t / 2$ & $1-t$ & & $1-s$ & 1 \\
Dominance & 1 & 1 & $1-t$ & & $1-s$ & 1 \\
Overdominance & $1-s$ & 1 & $1-t$ & & $1-s$ & $1-t$ \\
\cline { 1 - 2 } & & & &
\end{tabular}

of fitness space giving a stable polymorphism was smaller for haplodiploid loci. Allowing the intensity of selection and the correlation between male and female fitnesses to vary gave a similar result (Curtsinger, 1980; Pamilo and Crozier, 1981). The computer simulation studies done by Pamilo and Crozier (1981) showed that the average genic variation at the polymorphic loci was in most cases only slightly reduced in haplodiploids compared to diplodiploids, and in a few cases actually increased. However, because the probability of a polymorphism was less in haplodiploids, the overall average gene diversity was decreased. Balancing selection should therefore maintain fewer polymorphisms and a lower average heterozygosity in haplodiploid species compared to diplodiploid species.

A few additional complications arise when eusocial Hymenoptera are considered. For instance multiple mating by queens will increase the effective population size as will increasing the number of queens per colony (Wilson, 1963). Also, the production of males by workers is quite common in many species. Although hymenopteran workers are usually effectively sterile, under certain conditions they can undergo ovarian development and lay unfertilised eggs which develop into males. Worker-produced males occur in ants (Oster and Wilson, 1978; Cole, 1981 ; Plateaux, 1981), social wasps (Spradbery, 1973), bumble bees (Richards,
1946; Free, 1955; Pomeroy, 1979; van Honk, Velthius, Röseler and Hoogeveen, 1981; Owen and Plowright, 1982) and meliponid bees (Beig, 1972; Contel and Kerr, 1976).

In a previous paper (Owen, 1980) it was demonstrated that when there were workerproduced males a shift in the equilibrium gene frequency of a balanced polymorphism could occur. Moreover, for some fitness values a certain proportion of worker-produced males in the population would cause a polymorphism to be either extinguished or generated. However, an algebraic solution to the recurrence equations was not obtained. In this paper an analytical solution is given and for the general case the sufficient conditions are derived for there to be at least one nontrivial stable equilibrium. The three regular selection models considered by Lester (1975) and Pamilo (1979) are analysed in detail and the results used to examine the effect of worker-produced males on the probability of polymorphism and the expected genic variation.

\section{BALANCED POLYMORPHISMS WITH WORKER-PRODUCED MALES}

Consider a single biallelic locus and let the genotypes have relative fitnesses:

\begin{tabular}{|c|c|c|}
\hline & Female & \\
\hline$A_{1} A_{1}$ & $A_{1} A_{2}$ & $A_{2} A_{2}$ \\
\hline
\end{tabular}

Assume that initially the frequencies of the $A_{1}$ and $A_{1}$ alleles are respectively $p_{0}, q_{0}$ in the females and $P_{0}, Q_{0}$ in the males. In each generation let a proportion $\psi$ of the males be produced by the workers and $(1-\psi)$ by the queen in each colony. Assume also an infinite population size and random mating. In the next generation the gene frequencies will be, in the females

$$
\begin{aligned}
& p_{1}=\left[w_{11} p_{0} P_{0}+\frac{1}{2} w_{12}\left(p_{0} Q_{0}+q_{0} P_{0}\right)\right] / W_{0} \\
& q_{1}=\left[\frac{1}{2} w_{12}\left(p_{0} Q_{0}+q_{0} P_{0}\right)+w_{22} q_{0} Q_{0}\right] / W_{0}
\end{aligned}
$$

where

$$
W_{0}=w_{11} p_{0} P_{0}+w_{12}\left(p_{0} Q_{0}+q_{0} P_{0}\right)+w_{22} q_{0} Q_{0}
$$

and in the males

$$
\begin{aligned}
& P_{1}=\left[\left(1-\frac{1}{2} \psi\right) v_{1} p_{0}+\frac{1}{2} \psi v_{1} P_{0}\right] / V_{0} \\
& Q_{1}=\left[\left(1-\frac{1}{2} \psi\right) v_{2} q_{0}+\frac{1}{2} \psi v_{2} Q_{0}\right] / V_{0}
\end{aligned}
$$

where

$$
V_{0}=\left(1-\frac{1}{2} \psi\right)\left(v_{1} p_{0}+v_{2} q_{0}\right)+\frac{1}{2} \psi\left(v_{1} P_{0}+v_{2} Q_{0}\right) .
$$


Now at equilibrium the gene frequencies in each generation will be the same; therefore let $p_{0}=p_{1}=$ $p, P_{0}=P_{1}=P, W_{0}=W$ and $V_{0}=V$.

Substituting the equilibrium values $p, q, P, Q$ into equations (1) and (2) and dividing equation (1) by equation (2), gives

$$
\frac{p}{q}=\frac{\left(w_{11} p+\frac{1}{2} w_{12} q\right) P+\frac{1}{2} w_{12} p Q}{\left(w_{22} q+\frac{1}{2} w_{12} p\right) Q+\frac{1}{2} w_{12} q Q} .
$$

Rearranging and simplifying gives,

$$
\begin{aligned}
& P=\left[\left(w_{22}-\frac{1}{2} w_{12}\right) p q+\frac{1}{2} w_{12} p^{2}\right] / X \\
& Q=\left[\left(w_{11}-\frac{1}{2} w_{12}\right) p q+\frac{1}{2} w_{12} q^{2}\right] / X
\end{aligned}
$$

where

$$
X=\left(w_{11}-2 w_{12}+w_{22}\right) p q+\frac{1}{2} w_{12} .
$$

Similary from equations (4) and (5), at equilibrium

$$
\frac{P}{Q}=\frac{\left(1-\frac{1}{2} \psi\right) v_{1} P+\frac{1}{2} \psi v_{1} P}{\left(1-\frac{1}{2} \psi\right) v_{2} q+\frac{1}{2} \psi v_{2} Q}
$$

therefore after rearranging

$$
\frac{v_{2} q}{Q}-\frac{v_{1} p}{P}=\frac{\frac{1}{2} \psi\left(v_{1}-v_{2}\right)}{\left(1-\frac{1}{2} \psi\right)}
$$

Substituting equations (8) and (9) into equation (12) gives

$$
\begin{gathered}
\frac{X v_{2} q}{\left(w_{11}-\frac{1}{2} w_{12}\right) p q+\frac{1}{2} w_{12} q^{2}} \\
-\frac{X v_{1} p}{\left(w_{22}-\frac{1}{2} w_{12}\right) p q+\frac{1}{2} w_{12} p^{2}} \\
=\frac{\frac{1}{2} \psi\left(v_{1}-v_{2}\right)}{1-\frac{1}{2} \psi} .
\end{gathered}
$$

By putting $q=1-p$, multiplying out and rearranging, an equation for $p$ is obtained,

$$
\begin{aligned}
f(p)= & \left({ }_{2}^{1} \psi-1\right) A B p^{3}+\{A(B-C) \\
& \left.-\frac{1}{2} \psi\left[A(B-C)-D\left(v_{1}-v_{2}\right)\right]\right\} p^{2} \\
& +\left\{\left({ }_{2}^{1} w_{12} B+A C\right)-\frac{1}{2} \psi\left[\left(\frac{1}{2} w_{12} B+A C\right)\right.\right. \\
& \left.\left.+(D-E)\left(v_{1}-v_{2}\right)\right]\right\} p \\
& +\frac{1}{2} w_{12} C-\frac{1}{2} \psi\left[\frac{1}{2} w_{12} C+F\left(v_{1}-v_{2}\right)\right]=0
\end{aligned}
$$

where,

$$
\begin{aligned}
& A=\left(w_{11}-2 w_{12}+w_{22}\right) \\
& B=\left(w_{12}-w_{11}\right) v_{1}+\left(w_{12}-w_{22}\right) v_{2} \\
& C=w_{22} v_{2}-\frac{1}{2} w_{12}\left(v_{1}+v_{2}\right) \\
& D=\left(w_{12}-w_{11}\right)\left(w_{12}-w_{22}\right)
\end{aligned}
$$

$$
\begin{aligned}
& E=\frac{1}{2} w_{12}\left(w_{22}-w_{11}\right) \\
& F=\frac{1}{2} w_{12}\left(w_{22}-\frac{1}{2} w_{12}\right) .
\end{aligned}
$$

The root(s) of equation (14) give the equilibrium value(s) of $p$. The equilibrium gene frequency in males is obtained by substituting $p$ (and $q$ ) into equation (8).

Inspection of equation (14) shows that when there is no selection in the males (i.e., $v_{1}=v_{2}$ ) worker-produced males have no effect on the equilibrium gene frequency, which confirms Owen's (1980) finding. This is because the coefficient of each $p$ term is multiplied by the same constant $\left(1-\frac{1}{2} \psi\right)$, so the root remains unchanged.

More importantly, since $\boldsymbol{f}(p)$ is a third degree polynomial it can have up to three real roots, thus allowing the possibility of there being as many as three equilibria ( 2 stable, 1 unstable or 1 stable, 2 unstable) in $p[0,1]$. However the situation is a little unclear because when $\psi=0$ (the standard $X$ linked case) $\boldsymbol{f}(p)$, although simplified, remains cubic. Now it is well known that there can only be a single equilibrium (stable or unstable) in this case (Edwards, 1977). So it might be reasonable to think that the effect of worker-produced males would only be to shift the equilibrium gene frequencies and not produce additional roots. On the basis of mathematical continuity this will undoubtedly be true if $\psi$ is very small. However larger values of $\psi$ could lead to additional equilibria. Nevertheless, it is possible to obtain the sufficient conditions for there to be at least one stable equilibrium, and it will be shown that these conditions are exact for the three regular selection models (table 1) analysed by Pamilo (1979).

\section{Conditions for stable equilibrium}

To determine the conditions necessary for there to be an equilibrium the sign of the function $f(p)$, equation (14), is evaluated at $p=0$ and $p=1$. If the sign of $f(p)$ changes (from + to - or from to + ) in this interval then we know the shape of cubic polynomials that it has crossed the $x$-axis (satisfying $\boldsymbol{f}(p)=0$ ) either once (1 root) or thrice (3 roots).

Therefore, when $p=0$

$\boldsymbol{f}(0)=\frac{1}{2} w_{12}\left[w_{22} v_{2}-\frac{1}{2} \psi v_{1}\left(w_{22}-w_{12}\right)-\frac{1}{2} w_{12}\left(v_{1}+v_{2}\right)\right]$.

Now $f(0)<0$ if

$$
\frac{1}{2} w_{12}\left(v_{1}+v_{2}\right)>w_{22} v_{2}-\frac{1}{2} \psi v_{2}\left(w_{11}-w_{12}\right) .
$$

Similarly when $p=1$

$f(1)=\frac{1}{2} w_{12}\left[\frac{1}{2} w_{12}\left(v_{1}+v_{2}\right)-w_{11} v_{1}+\frac{1}{2} \psi v_{2}\left(w_{11}-w_{12}\right)\right]$. 
Now $f(1)>0$ if

$$
\frac{1}{2} w_{12}\left(v_{1}+v_{2}\right)>w_{11} v_{1}-\frac{1}{2} \psi v_{2}\left(w_{11}-w_{12}\right) .
$$

So that the conditions for there to be at least one polymorphic equilibrium when there are workerproduced males are,

$$
{ }_{2}^{1} w_{12}\left(v_{1}+v_{2}\right)>\left\{\begin{array}{l}
w_{11} v_{1}-\frac{1}{2} \psi v_{2}\left(w_{11}-w_{12}\right) \\
w_{22} v_{2}-\frac{1}{2} \psi v_{1}\left(w_{22}-w_{12}\right) .
\end{array}\right.
$$

Note that when $\psi=0$ the conditions reduce to the standard ones, $w_{11} v_{1}<\frac{1}{2} w_{12}\left(v_{1}+v_{2}\right)>w_{22} v_{2}$ (Haldane, 1926; Bennett, 1958; Mandel, 1959; Edwa:ds, 1977).

Some general observations can be made; with overdominance $\left(w_{12}>w_{11}, w_{12}\right)$ or dominance $\left(w_{11}=w_{12}>w_{22}\right)$ in the females the conditions are more stringent than when $\psi=0$. On the other hand with codominance $\left(w_{11}>w_{12}>w_{22}\right)$ whether the conditions are more or less stringent depends on the relative fitness values and the value of $\psi$.

Endpoint analysis shows that when the conditions (equation 15) are satisfied then at least one of the equilibria will be stable. If $p$ and $P$ are small we have approximately, $W=w_{22}, V=v_{2}$,

$$
\begin{aligned}
& p_{1}=\frac{w_{12}}{2 w_{22}}\left(p_{0}+P_{0}\right) \\
& P_{1}=v_{1}\left[\left(1-\frac{1}{2} \psi\right) p_{0}+\frac{1}{2} \psi P_{0}\right] .
\end{aligned}
$$

The corresponding eigenvalue equation is

where

$$
\lambda^{2}-L \lambda-M=0
$$

and

$$
L=\frac{w_{12}}{2 w_{22}}+\frac{\psi v_{1}}{2 v_{2}}
$$

$$
\begin{aligned}
M & =(-)\left|\begin{array}{cc}
\frac{w_{12}}{2 w_{22}}, & \frac{w_{12}}{2 w_{22}} \\
\frac{\left(1-\frac{1}{2} \psi\right) v_{1}}{v_{2}}, & \frac{\psi v_{1}}{2 v_{2}}
\end{array}\right| \\
& =\frac{w_{12} v_{1}}{2 w_{22} v_{2}}(1-\psi) .
\end{aligned}
$$

It is known (Edwards, 1977) that a necessary condition for both eigenvalues to be less than unity is $L+M<1$. Therefore $L+M>1$ is a sufficient condition for the fixation point $p_{0}=P_{0}=0$ to be unstable, implying that $p$ and $P$ will increase in frequency. The condition is:

$$
\frac{w_{12}}{2 w_{22}}+\frac{\psi v_{1}}{2 v_{2}}+\frac{w_{12} v_{1}}{2 w_{22} v_{2}}(1-\psi)>1
$$

i.e.,

$$
\frac{1}{2} w_{12}\left(v_{1}+v_{2}\right)>w_{22} v_{2}-\frac{1}{2} \psi v_{1}\left(w_{22}-w_{12}\right) .
$$

Similarly the sufficient condition for the fixation point $q_{0}=Q_{0}=0$ to be unstable is,

$$
\frac{1}{2} w_{12}\left(v_{1}+v_{2}\right)>w_{11} v_{1}-\frac{1}{2} \psi v_{2}\left(w_{11}-w_{12}\right) \text {. }
$$

Proceeding heuristically we expect these to be sufficient conditions for at least one of the nontrivial equilibria to be stable.

In some cases $f(p)$, equation (14) may have three real roots between 0 and 1 . It is wrong to conclude, however, that all of the roots will necessarily correspond to real gene frequency equilibria. This is because the male and female gene frequencies are not independent. The male equilibrium gene frequencies are obtained from equations (8) and (9) where $p$ (with corresponding $q)$ is a root of $f(p)$. Clearly for certain values of $p$ and $q$ and the female fitnesses, $P$ and $Q$ will lie outside the permissible range, i.e., $0 \leqq P, Q \leqq 1$. Thus a permissible root of $f(p)$ will not, in fact, always give rise to a "real" gene frequency equilibrium. Therefore for the general case with five independent viabilities it is not possible to give any general rules to determine the exact number of equilibria. Nevertheless it does follow from the endpoint analysis that, provided the conditions are satisfied, there will always be at least one stable equilibrium with $0<P<1$.

\section{Equilibrium gene frequencies}

To find the equilibrium gene frequencies necessitates determining the roots of the cubic equation $f(p)$ which can be done using entirely standard algebraic methods (Borofsky, 1950).

Write equation (14) in the form

$$
p^{3}+a p^{2}+b p+c=0
$$

where,

$$
\left.\begin{array}{rl}
a= & \left\{A(B-C)-{ }_{2}^{1} \psi[A(B-C)\right. \\
& \left.\left.-D\left(v_{1}-v_{2}\right)\right]\right\} /\left(\frac{1}{2} \psi-1\right) A B \\
b= & \left\{\left(\frac{1}{2} w_{12} B+A C\right)-\frac{1}{2} \psi\left[\left(\frac{1}{2} w_{12} B+A C\right)\right.\right. \\
& \left.\left.+(D-E)\left(v_{1}-v_{2}\right)\right]\right\} /\left(\frac{1}{2} \psi-1\right) A B \\
c= & \left\{\frac{1}{2} w_{12} C-\frac{1}{2} \psi\left[\frac{1}{2} w_{12} C\right.\right. \\
& \left.\left.+F\left(v_{1}-v_{2}\right)\right]\right\} /\left(\frac{1}{2} \psi-1\right) A B .
\end{array}\right\}
$$

Also,

$$
3 \alpha=b-\left(a^{2} / 3\right)
$$

and,

$$
2 \beta=\frac{2 a^{3}}{27}-\frac{a b}{3}+c .
$$


Solutions:

(1) $\beta^{2}+\alpha^{3} \geqq 0$ then there is one real and two conjugate complex roots (Borofsky, 1950). Only the real root need be considered. From Cardan's formula,

and

$$
\begin{aligned}
& y_{1}=\sqrt[3]{-\beta+\sqrt{\beta^{2}+\alpha^{3}}} \\
& y_{2}=\sqrt[3]{-\beta-\sqrt{\beta^{2}+\alpha^{3}}}
\end{aligned}
$$

$$
p=y_{1}+y_{2}-(a / 3) .
$$

(2) If $\beta^{2}+\alpha^{3}<0$ then there are three real and different solutions (Borofsky, 1959). A trigonometrical method is used;

$$
\cos \phi=\frac{-\beta}{+\sqrt{-\alpha^{3}}}
$$

and

$$
\begin{aligned}
& p_{1}=2 \sqrt{-\alpha} \cos \frac{\phi}{3}-(a / 3) \\
& p_{2}=-2 \sqrt{-\alpha} \cos \left(\frac{\phi}{3}+60^{\circ}\right)-(a / 3) \\
& p_{3}=-2 \sqrt{-\alpha} \cos \left(\frac{\phi}{3}-60^{\circ}\right)-(a / 3) .
\end{aligned}
$$

The male gene frequencies in both cases are calculated from equations (8) and (9).

To summarise the results for the general case: sufficient conditions for there to be at least one stable equilibrium have been derived. This of course does not exclude the existence of other stable equilibria when these conditions are not satisfied. However, all equilibrium gene frequencies for any given set of fitness values can be found as they are given by the roots of the cubic equation $f(p)$. The stability of any particular equilibrium can be tested using formula (23) in Owen (1980).
ANALYSIS OF REGULAR SELECTION MODELS

The general case will not be pursued further here, rather the three regular selection models examined by Lester (1975) and Pamilo (1979) will be analysed in detail. The parameterisation used (table 1) allows the five fitnesses to be described by two variables $s$ and $t$ (both with values from zero to one) so the range of permissible fitness values can be represented in a two-dimensional space (fig. 1).

\section{Codominance}

Conditions for equilibrium are obtained by substituting the parameterised fitnesses into equation (15), which gives:

$$
\left\{\begin{array}{l}
t<\frac{2 s}{2-s-\psi} \\
s<\frac{2\left(1-\frac{1}{2} \psi\right) t}{2-(1+\psi) t} .
\end{array}\right.
$$

The regions of stable polymorphic equilibria, for various values of $\psi$, are contained between the relevant pairs of curves shown in fig. la.

Equilibrium gene frequencies; equation (14) reduces to a quadratic

$$
k p^{2}+l p+m=0
$$

where

$$
\begin{aligned}
k & =2 t \psi \\
l & =(4-2 t)-(3 t-2) \psi \\
m & =\left[\frac{4}{t}-\frac{4}{s}+\frac{2 t}{s}-4+t\right]-\left[\frac{t}{s}-\frac{2}{s}+2-t\right] \psi
\end{aligned}
$$

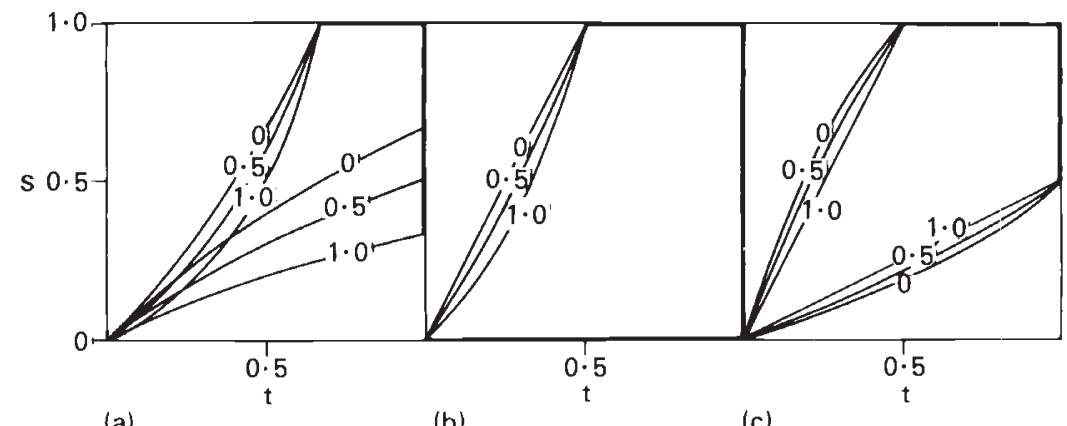

(a)

(b)

(c)

Figure 1 The region of permissible fitness values defined by the selection coefficients $s$ and $t$ for the (a) Codominance (b) Dominance and (c) Overdominance models. The areas of stable equilibria are contained between the curves shown for various values of $\psi$. See Pamilo (1979) for the corresponding areas for autosomal (diplodiploid) loci. 
This has one positive and one negative root, of which only the former is of interest i.e.,

$$
p=\frac{-l+\sqrt{l^{2}-4 k m}}{2 k}
$$

and, from equation (8),

$$
P=\frac{(2-3 t) p q}{(2-t)}+p^{2} .
$$

Note that because equation (18) has only one relevant root there can be at most only one equilibrium and it follows that the conditions are both sufficient and necessary for a stable polymorphism. No stable equilibria can exist if the conditions are not satisfied.

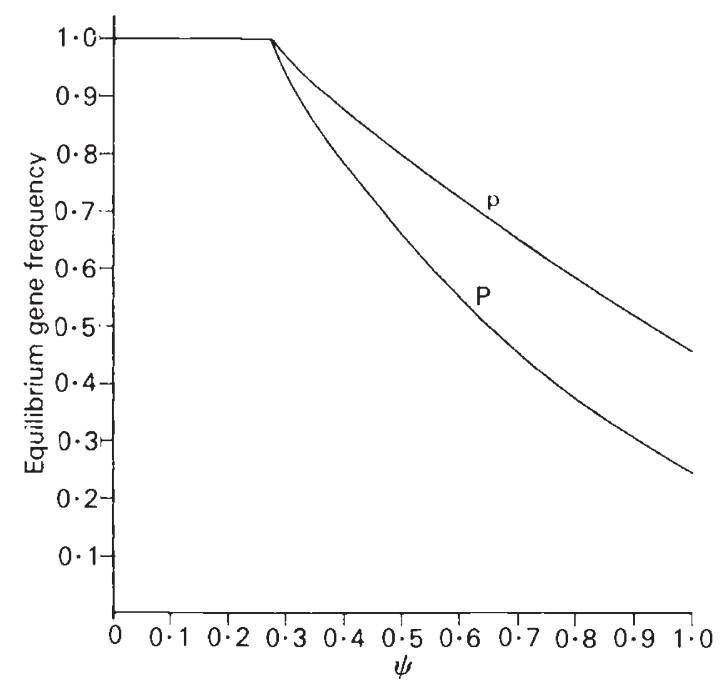

Figure 2 An example of the effect that worker-produced males have on the existence of a polymorphism and on the female $(p)$ and male $(P)$ equilibrium gene frequencies. The fitnesses are $w_{11}=1 \cdot 0, w_{12}=0 \cdot 7, w_{22}=0 \cdot 3, v_{1}=0 \cdot 6, v_{2}=1 \cdot 0$, i.e., codominance with $s=0 \cdot 4, t=0.6$. The conditions for equilibrium are satisfied when $\psi>0 \cdot 27$.

Fig. 2 shows an example of the effect of workerproduced males on the equilibrium gene frequencies when there is codominance.

\section{Dominance}

Condition for stable equilibrium (from equation $(15)$,

$$
s<\frac{2(1-\psi) t}{1-\psi t}
$$

which for each value of $\psi$ defines the regions shown in fig. $1 b$.
Equilibrium gene frequencies; in this case the roots of a cubic equation (16) must be found. Substituting the parameterised fitness into equations (17) gives,

$$
\begin{aligned}
& a=\frac{s}{2 t}-2 \\
& b=1+\frac{(1-s)}{2 t}+\frac{\psi s}{(4-2 \psi) t} \\
& c=s / 4 t^{2}-\frac{1}{2 t}-\left(\frac{1}{2} t-\frac{1}{4}\right) s /(2-\psi) t^{2} .
\end{aligned}
$$

It was found that $\beta^{2}+\alpha^{3}>0$ for all relevant values of $s, t$ and $\psi$ i.e., $0 \leqq s, t, \psi \leqq 1$. This means that for the dominance model the equation $f(p)$ has only one real root thus a single equilibrium. Again the condition is therefore both sufficient and necessary.

Therefore,

$$
p=y_{1}+y_{2}-(a / 3)
$$

where $y_{1}$ and $y_{2}$ were given earlier, and

$$
P=\frac{\left(\frac{1}{2}-t\right) p q+\frac{1}{2} p^{2}}{\frac{1}{2}-t p q} \text {. }
$$

An example will illustrate the calculations involved. For instance let $t=0 \cdot 5, s=0 \cdot 6, \psi=0 \cdot 5$. Then $a=1.4, b=1.6, c=-0.4$. Therefore $\alpha=0.31555$ and $\beta=0.071705$. Thus $\beta^{2}+\alpha^{3}=0.0365614$, which is positive, therefore there is only one real root and Cardan's formula can be used, giving $p=0.31296$ and $P=0 \cdot 12477$.

\section{Overdominance}

The conditions for stable equilibria, from equation (15) are,

$$
\left\{\begin{array}{l}
t<\frac{3 s-2 s^{2}-\psi s}{1-\psi s} \\
s<\frac{3 t-2 t^{2}-\psi t}{1-\psi t}
\end{array}\right.
$$

The region defined by these two inequalities is shown in fig. lc, for various values of $\psi$.

Equilibrium gene frequencies; in this case also the cubic equation must be solved. The equations (17) do not easily simplify when the parameterised fitness values are substituted in them. Instead $a, b$ and $c$ have to be calculated directly using equations (14) and (17). For most values of $s, t$ and $\psi$ the polynomial $f(p)$ has only one real root but in a few cases it has three real and different roots when the conditions for stable equilibrium are satisfied. An example of each follows. 


\section{One root}

If $s=0.4, t=0.7$ and $\psi=0.5$ then the conditions for equilibrium are satisfied (fig. 1c).

Therefore,

$$
\begin{aligned}
& a=-1.7434, \quad b=1 \cdot 1677, \quad c=-0.3434 \\
& \alpha=0.51496, \quad \beta=-0.028691
\end{aligned}
$$

and

$$
\beta^{2}+\alpha^{3}=0 \cdot 0009596
$$

positive so there is a single real root with $p=$ 0.84013 and $P=0.92560$.

\section{Three roots}

If $s=0 \cdot 6, t=0.99$ and $\psi=1.0$ then conditions for equilibrium are satisfied (fig. $1 \mathrm{c}$ ).

Therefore,

$$
\begin{aligned}
& a=-1 \cdot 2369, \quad b=0.36997, \quad c=-0.017365 . \\
& \alpha=-0.0500018, \quad \beta=-0.004562
\end{aligned}
$$

and

$$
\beta^{2}+\alpha^{3}=-0 \cdot 000104
$$

negative so there are three real roots, and the trigonometrical solution is used. Thus

$$
\begin{aligned}
\cos \phi & =0.004562 / \sqrt{0.000125}=0.4080391, \\
\phi & =65.9^{\circ}, \quad \phi / 3=21.97^{\circ}
\end{aligned}
$$

and

$$
\cos (\phi / 3)=0 \cdot 9278
$$

Therefore, $p_{1}=0.8272, P_{1}=0.9976 ; p_{2}=0.3500$ but $P_{2}=-0.3632 ;$ and $p_{3}=0.0604$ but $P_{3}=$ -0.06339 .

The last two roots clearly give inadmissible values for the male gene frequencies $P_{2}$ and $P_{3}$, so these roots do not correspond to real gene frequency equilibria. Iteration of the recurrence equations (1), (2), (3), (4), (5) and (6) for this example (fig. 3) indeed shows that only the first root corresponds to an equilibrium with gene frequencies $p_{1}$ and $P_{1}$. The reason for this was pointed out earlier, namely that the male and female gene frequencies are not independent. $P$ and $Q$ must both be greater or equal to zero and this constrains $p$ and $q$ to be within a given range.

Require

$$
P=\frac{\left[\left(w_{22}-\frac{1}{2} w_{12}\right) p q+\frac{1}{2} w_{12} p^{2}\right]}{\left(w_{11}+w_{22}-2 w_{12}\right) p q+\frac{1}{2} w_{12}} \geqq 0
$$

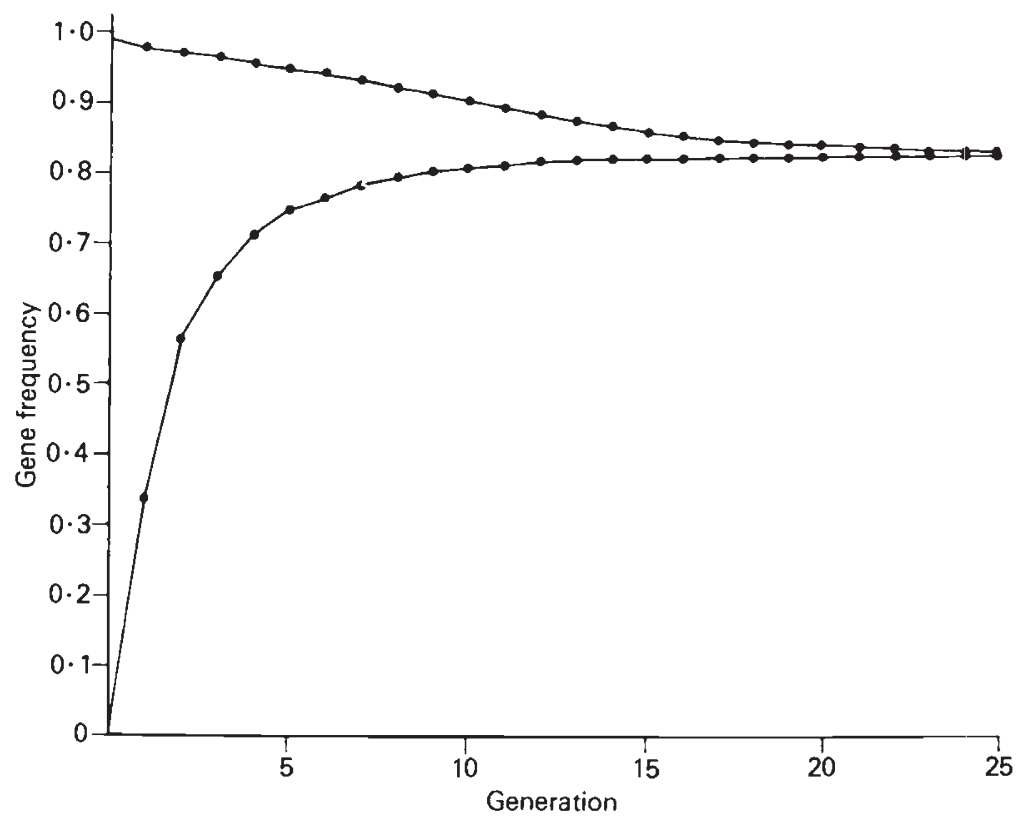

Figure 3 Gene frequency trajectories obtained by iterating the gene frequency recurrence equations for overdominance with $s=0 \cdot 6$, $t=0.99$ and $\psi=1 \cdot 0$. The same, and single, equilibrium gene frequency is approached from any initial gene frequency. Only the female gene frequency, $p$, is shown for each generation 
i.e.,

$$
\begin{gathered}
\left(w_{22}-\frac{1}{2} w_{12}\right)\left(p-p^{2}\right)+\frac{1}{2} w_{12} p^{2} \geqq 0 \\
p\left[\left(w_{22}-\frac{1}{2} w_{12}\right)+\left(w_{12}-w_{22}\right) p\right] \geqq 0
\end{gathered}
$$

therefore

$$
p \geqq\left({ }_{2}^{1} w_{12}-w_{22}\right) /\left(w_{12}-w_{22}\right) .
$$

Similarly

$$
q \geqq\left(\frac{1}{2} w_{12}-w_{11}\right) /\left(w_{12}-w_{11}\right) .
$$

For the overdominance model the above become,

$$
\begin{aligned}
& p \geqq \frac{t-\frac{1}{2}}{t} \\
& q \geqq \frac{s-\frac{1}{2}}{s} .
\end{aligned}
$$

So for the example given above we require,

$$
p \geqq \frac{0.99-0.5}{0.99} \geqq 0.4949 ; \quad q \geqq \frac{0 \cdot 6-0.5}{0.6} \geqq 0 \cdot 1667 \text {. }
$$

If can be seen that both these conditions are satisfied only by the first root. Only a small proportion of the polynomials have three roots (see table 3 ) and no examples were found which gave more than one equilibrium. Thus it seems reasonable to conjecture that this is always true. However it does not seem possible to prove this.

In the regions in fig. $1 \mathrm{c}$ where the equilibrium conditions, inequalities (23a) and (23b), are not satisfied it was found that a very small proportion of the polynomials $\boldsymbol{f}(p)$ had two roots between 0 and 1 . In these regions $f(0)$ and $f(1)$ are either both positive or both negative. Since $f(0)$ and $f(1)$ are the same sign it means that there are either no roots or two roots. Two roots occurred only when $\psi \geqq 0.99$ and when either $s$ or $t$ was extreme e.g., 0.99 , and this was rare. For $\psi=1.0$ only 128 out of 4912 ( 2.6 per cent $)$ polynomials examined had two roots. Also no examples were found where the roots corresponded to a real gene frequency equilibrium i.e., the inequalities (24a) and (24b) were not both satisfied. Again a reasonable conjecture, difficult to prove, is that in these regions no stable equilibria occur even when $\boldsymbol{f}(p)$ has two roots.

To summarise; for the overdominance model the conditions for equilibrium are both sufficient and necessary, at least for $\psi<0.99$ and in fact appear to be so for all values of $\psi$.

\section{PROBABILITY OF A POLYMORPHISM}

Fig. 1 shows the regions in the two-dimensional fitness space in which the conditions for stable polymorphic equilibria are satisfied when there is either Codominant, Dominant or Overdominant selection (table 1). The selection coefficient $s$ and $t$, which define the fitness space, are assumed to be independent and rectangularly distributed (Pamilo, 1979). The areas of the regions depend on the value of $\psi$. The probability of a polymorphism can be quantified by calculating the areas of these regions using integral calculus (see Appendix). Pamilo (1979) used this method to compare the probability of a polymorphism at $X$-linked (equivalent to $\psi=0$ ) and autosomal loci for these three regular selection models. For comparison with Pamilo's results table 2 gives the ratio of the

\begin{tabular}{|c|c|c|c|c|c|c|c|c|c|c|}
\hline \multirow[b]{2}{*}{$\psi$} & \multirow{2}{*}{$\begin{array}{l}\text { Selection } \\
\text { coeff. } \\
\text { range: }\end{array}$} & \multicolumn{3}{|c|}{ Codominance } & \multicolumn{3}{|c|}{ Dominance } & \multicolumn{3}{|c|}{ Overdominance } \\
\hline & & $0-1 \cdot 0$ & $0-0 \cdot 125$ & $0-0 \cdot 01$ & $0-1 \cdot 0$ & $0-0 \cdot 125$ & $0-0.01$ & $0-1 \cdot 0$ & $0-0 \cdot 125$ & $0-0.01$ \\
\hline 0 & & 0.631 & 0.522 & 0.496 & 0.750 & 0.750 & 0.750 & 0.583 & 0.660 & 0.666 \\
\hline $0 \cdot 1$ & & 0.665 & 0.547 & 0.498 & 0.746 & 0.738 & 0.737 & 0.577 & 0.649 & 0.655 \\
\hline $0 \cdot 2$ & & 0.699 & 0.548 & 0.493 & 0.741 & 0.725 & 0.722 & 0.570 & 0.637 & 0.642 \\
\hline 0.3 & & 0.732 & 0.544 & 0.487 & 0.736 & 0.710 & 0.706 & 0.563 & 0.624 & 0.629 \\
\hline 0.4 & & 0.764 & 0.537 & 0.478 & 0.731 & 0.694 & 0.688 & 0.556 & 0.611 & 0.615 \\
\hline 0.5 & & 0.795 & 0.527 & 0.468 & 0.726 & 0.676 & 0.667 & 0.548 & 0.596 & 0.600 \\
\hline 0.6 & & 0.826 & 0.514 & 0.454 & 0.720 & 0.655 & 0.644 & 0.539 & 0.580 & 0.583 \\
\hline 0.7 & & 0.855 & 0.498 & 0.438 & 0.714 & 0.632 & 0.617 & 0.531 & 0.562 & 0.565 \\
\hline 0.8 & & 0.882 & 0.479 & 0.420 & 0.708 & 0.605 & 0.585 & 0.521 & 0.543 & 0.545 \\
\hline 0.9 & & 0.908 & 0.457 & 0.399 & 0.701 & 0.574 & 0.548 & 0.511 & 0.523 & 0.524 \\
\hline $1 \cdot 0$ & & 0.933 & 0.432 & 0.375 & 0.693 & 0.538 & $0 \cdot 503$ & 0.500 & 0.500 & $0 \cdot 500$ \\
\hline
\end{tabular}

Table 2 The ratio of the probability of a polymorphism at a haplodiploid locus to the probability at an autosomal (diplodiploid) locus. The haplodiploids are social Hymenoptera with a proportion $\psi$ of the males produced by the workers. The selection coefficients $s$ and $t$, are assumed to be rectangularly distributed and independent of each other (fig. 1). The probability of a polymorphism is defined as the area of the fitness space in which the conditions (equation 15) for stable equilibria are satisfied (see section 3 and the Appendix). For each selection model the ratios were calculated for three ranges of selection coefficients; $0-1 \cdot 0,0-0 \cdot 125$ and $0-0 \cdot 01$ 
area of the fitness region giving polymorphic equilibria to that in diplodiploids for eleven values of $\psi$ and for three ranges of selection coefficients.

Codominance When the range of selection coefficients is $0-1.0$ the effect of increasing the proportion of worker-produced males is to increase the probability of a polymorphism substantially, although it is always less than in diplodiploids. However, the picture is different when the selection differentials are smaller. Initially the ratio increases slightly as $\psi$ increases, but then the trend is reversed and the probability of a polymorphism is reduced.

Dominance and Overdominance For all ranges of selection coefficients an increase in $\psi$ leads to a decrease in the probability of a polymorphism. The percentage decrease is greater the smaller the range of selection coefficients.

\section{EXPECTED GENIC VARIATION}

The genic variation, averaged over all stable polymorphic equilibria, was calculated for eleven values of $\psi$ and three ranges of selection coefficient for each of the selection models (table 3 ). The fitnesses used to calculate the gene frequencies were derived by letting $s$ and $t$ vary from zero to $x$ with intervals of $x / 100$ where $x$ is the upper value of selection coefficient allowed i.e., 1 $0,0 \cdot 125$ or 0.01 . The equations derived for each selection model were used to calculate the equilibrium gene frequencies. In the overdominance model for the

Table 3 The average genic variation $\bar{H} p$ at $N$ stable polymorphic equilibria for each of three ranges of selection coefficients for the three selection models. In the overdominance model a small number (given in parentheses) of the polynomials had more than one root. $\bar{H} p$ was, however, calculated only for the polynomials with one root which could be solved easily using Cardan's formula

\begin{tabular}{|c|c|c|c|c|c|c|c|}
\hline \multirow[b]{2}{*}{ Model } & \multirow[b]{2}{*}{$\psi$} & \multicolumn{2}{|c|}{$0-1 \cdot 0$} & \multicolumn{3}{|c|}{$\begin{array}{l}\text { Range of Selection Coefficients } \\
0-0 \cdot 125\end{array}$} & -0.01 \\
\hline & & $N$ & $\overrightarrow{H p}$ & $N$ & $\bar{H} p$ & $N$ & $\bar{H} p$ \\
\hline \multirow[t]{11}{*}{ Codominance } & 0 & 2468 & $0 \cdot 354$ & 420 & $0 \cdot 350$ & 100 & 0.500 \\
\hline & $0 \cdot 1$ & 2603 & $0 \cdot 352$ & 436 & 0.325 & 35 & 0.326 \\
\hline & $0 \cdot 2$ & 2733 & $0 \cdot 354$ & 418 & 0.338 & 35 & $0 \cdot 346$ \\
\hline & $0 \cdot \overline{3}$ & 2867 & $0 \cdot 354$ & 421 & 0.333 & 32 & $0 \cdot 351$ \\
\hline & 0.4 & 2992 & 0.355 & 412 & $0 \cdot 337$ & 37 & 0.381 \\
\hline & 0.5 & 3111 & $0 \cdot 356$ & 410 & 0.333 & 40 & $0 \cdot 380$ \\
\hline & 0.6 & 3228 & 0.357 & 400 & $0 \cdot 331$ & 33 & $0 \cdot 343$ \\
\hline & 0.7 & 3345 & $0 \cdot 357$ & 385 & $0 \cdot 332$ & 28 & 0.358 \\
\hline & 0.8 & 3455 & $0 \cdot 357$ & 374 & $0 \cdot 332$ & 35 & $0 \cdot 365$ \\
\hline & 0.9 & 3556 & 0.359 & 355 & $0 \cdot 331$ & 25 & $0 \cdot 364$ \\
\hline & $1 \cdot 0$ & 3654 & $0 \cdot 358$ & 340 & $0 \cdot 338$ & 50 & 0.444 \\
\hline \multirow[t]{11}{*}{ Dominance } & 0 & 7527 & $0 \cdot 348$ & 7527 & $0 \cdot 353$ & 7512 & 0.354 \\
\hline & $0 \cdot 1$ & 7478 & $0 \cdot 350$ & 7403 & 0.354 & 7349 & 0.354 \\
\hline & 0.2 & 7438 & $0 \cdot 352$ & 7270 & $0 \cdot 355$ & 7250 & 0.355 \\
\hline & $0 \cdot 3$ & 7389 & $0 \cdot 354$ & 7124 & 0.355 & 7084 & 0.355 \\
\hline & 0.4 & 7339 & $0 \cdot 356$ & 6958 & $0 \cdot 354$ & 6901 & 0.354 \\
\hline & 0.5 & 7287 & $0 \cdot 357$ & 6778 & $0 \cdot 353$ & 6700 & 0.352 \\
\hline & 0.6 & 7233 & $0 \cdot 350$ & 6567 & 0.351 & 6455 & $0 \cdot 350$ \\
\hline & $0 \cdot 7$ & 7170 & $0 \cdot 360$ & 6331 & $0 \cdot 348$ & 6180 & 0.347 \\
\hline & 0.8 & 7105 & $0 \cdot 362$ & 6062 & $0 \cdot 344$ & 5862 & $0 \cdot 343$ \\
\hline & 0.9 & 7037 & $0 \cdot 363$ & 5752 & $0 \cdot 339$ & 5485 & 0.338 \\
\hline & $1 \cdot 0$ & 6957 & $0 \cdot 364$ & 5392 & $0 \cdot 335$ & 5050 & $0 \cdot 335$ \\
\hline \multirow[t]{11}{*}{ Overdominance } & 0 & 5888 & $0 \cdot 354$ & 6660 & $0 \cdot 366$ & 6700 & $0 \cdot 367$ \\
\hline & $0 \cdot 1$ & 5818 & $0 \cdot 353$ & 6554 & $0 \cdot 365$ & 6610 & 0.365 \\
\hline & $0 \cdot 2$ & 5752 & $0 \cdot 352$ & 6436 & $0 \cdot 364$ & 6486 & $0 \cdot 364$ \\
\hline & $0 \cdot 3$ & $5680(2)$ & $0 \cdot 350$ & 6308 & $0 \cdot 363$ & 6354 & $0 \cdot 363$ \\
\hline & 0.4 & $5606(6)$ & 0.348 & 6166 & $0-362$ & 6208 & $0 \cdot 363$ \\
\hline & 0.5 & $5520(10)$ & 0.346 & 6014 & $0 \cdot 361$ & 6040 & $0 \cdot 363$ \\
\hline & 0.6 & $5420(18)$ & $0 \cdot 344$ & 5854 & $0 \cdot 360$ & 5884 & $0 \cdot 361$ \\
\hline & 0.7 & $5320(36)$ & $0 \cdot 341$ & 5680 & $0 \cdot 359$ & 5704 & $0 \cdot 360$ \\
\hline & 0.8 & $5186(80)$ & 0.338 & 5488 & $0 \cdot 358$ & 5500 & $0 \cdot 350$ \\
\hline & 0.9 & $4984(178)$ & 0.335 & 5282 & $0 \cdot 356$ & 5288 & $0 \cdot 358$ \\
\hline & $1 \cdot 0$ & $4728(342)$ & 0.333 & 5054 & $0 \cdot 354$ & 5030 & $0 \cdot 358$ \\
\hline
\end{tabular}


selection range $0-1 \cdot 0$ gene frequencies were calculated only if the polynomial had one real root which was true in 98.9 per cent $(50,902$ out of $60,574)$ of the cases. The genic variation or genic diversity for haplodiploids (Pamilo, 1979) is defined as $\bar{H} p=2 \bar{p}(1-\bar{p})$ where $\bar{p}=(2 p+P) / 3$, the average allele frequency. The values of $\bar{H} p$ calculated and the number of equilibria examined are given in table 3 .

Codominance With selection coefficients in the range $0-1 \cdot 0 \bar{H} p$ increases, but only very slightly (up to $1 \cdot 3$ per cent when $\psi=1 \cdot 0$ ), with increasing $\psi$. With selection differentials of $0-0 \cdot 125$ there is a corresponding slight decrease. When the selection coefficient range is very small, $0-0 \cdot 01, \bar{H} p$ decreases by about 35 per cent when $\psi=0 \cdot 1$. It then increases as $\psi$ increases but fluctuates about 24 per cent-30 per cent below the value at $\psi=0$. When all the males are worker-produced $(\psi=1 \cdot 0)$ the reduction in $\bar{H} p$ is the least at about 11 per cent.

Dominance Again, in the selection coefficient range $0-1 \cdot 0 \bar{H} p$ steadily increases as the proportion of worker-produced males increases. The maximum increase (at $\psi=1.0$ ) is however only 4.4 per cent. When the selection differentials are smaller $(0-0 \cdot 125$ and $0-0 \cdot 01)$ there is at first a slight increase in $\bar{H} p$, then for $\psi>0.4$ it steadily decreases.

Overdominance For all ranges of selection coefficients worker-produced males cause a small decrease (maximum 6 per cent) in $\bar{H} p$.

In conclusion, worker-produced males at most can cause a slight increase in the average genic variation at polymorphic loci. In most cases a reduction in $\bar{H} p$ is expected, this may be small though a large reduction can occur with codominance and weak selection.

\section{DISCUSSION}

The recurrence equations for a balanced polymorphism in social Hymenoptera when there are worker-produced males were solved. The equilibrium gene frequencies are given by the roots of a cubic equation. The effect of the workerproduced males is to shift the female and male equilibrium gene frequencies and may cause a polymorphism to be extinguished or generated. These effects only occur if there is a selective difference between the male genotypes. The sufficient conditions for there to be at least one stable equilibrium were derived for the general case of five independent fitnesses. It was shown that these conditions were both necessary and sufficient to describe all stable equilibria in the three regular selection models analysed in detail.

The effect of worker-produced males with dominant and overdominant selection is to reduce the probability of a polymorphism, in some cases quite substantially (e.g., 33 per cent with dominance, $\psi=1 \cdot 0$, selection $0-0 \cdot 01$ ) - see table 2 .

With codominance the selection intensity is important. If the selection coefficients range from $0-1.0$ then worker-produced males increase the probability of a polymorphism up to a maximum of 32 per cent when $\psi=1 \cdot 0$. Even this however, is still less than in comparable diplodiploids. On the other hand with lower selection intensities only a small increase occurs with low values of $\psi$, while higher values lead to a decrease (table 2).

Worker-produced males also affect the average genic variation $(H p)$ at polymorphic loci. A slight increase with $\psi$ occurs in the codominance and dominance models when the selection coefficients range from $0-1 \cdot 0$. However, in all models a decrease occurs with increasing $\psi$ at lower selection intensities. This is particularly severe with codominance (table 3 ).

Consequently we can conclude that the effect of worker-produced males generally will be to reduce the number of polymorphisms maintained and their average genic variability compared to diplodiploid species. Even in the most favourable case, when the loci have codominant fitnesses and selection coefficients from $0-1 \cdot 0$, the probability of a polymorphism will still be less in the haplodiploid species.

Compared to other Hymenoptera, species with worker-produced males will suffer a reduced frequency of polymorphisms and lower mean genic diversity at loci with dominant and overdominant fitnesses. In contrast at loci with codominant fitnesses the frequency of polymorphisms and genic diversity will be higher if the selective differentials are large $(0-1 \cdot 0)$. Therefore, the overall level of genic variation depends on the proportion of codominant loci (with respect to fitness) and the intensity of selection.

For convenience it was assumed that the value of $\psi$ is the same in each colony. In fact this assumption is not essential because for individual, as opposed to colony-level selection, and random mating, it is equivalent to using the mean value of $\psi$ in the population. Certainly in practice the value of $\psi$ does vary from colony to colony (Owen and Plowright, 1982). More importantly $\psi$ may vary from year to year either stochastically or regularly. 
This variation might have some interesting consequences. Oscillations around a mean equilibrium gene frequency are almost bound to occur. The conditions for equilibrium would be altered and might depend, say, on the geometric mean of $\psi$ over time rather than on the arithmetic mean.

\section{APPENDIX}

The areas of stable equilibria were calculated using the formulae derived below which reduce to those given by Pamilo (1979) as $\psi \rightarrow 0$. The areas of stable equilibria at autosomal (diplodiploid) loci were calculated using the formulae derived by Pamilo. Table 2 gives the ratio of the area of stable equilibria in haplodiploids to that in diplodiploids. The results were checked by numerical integration using Simpson's rule with six strips. The areas of stable polymorphic equilibria $(T)$ are,

\section{Codominance model}

$$
T=x^{2}-(T 1+T 2)
$$

(i) For $0 \leqq s, t \leqq x$

$$
\begin{aligned}
T 1= & x t(x)-\int_{0}^{t(x)} \frac{2\left(1-\frac{1}{2} \psi\right) t}{2-(1+\psi) t} d t \\
= & x t(x)+\frac{2\left(1-\frac{1}{2} \psi\right) t(x)}{1+\psi} \\
& +\frac{4\left(1-\frac{1}{2} \psi\right)}{(1+\psi)^{2}} \ln \left[1-\frac{1}{2}(1+\psi) t(x)\right]
\end{aligned}
$$

where

$$
t(x)=\frac{2 x}{2\left(1-\frac{1}{2} \psi\right)+(1+\psi) x}
$$

(ii) For $0 \leqq t, s \leqq x$

$$
\begin{aligned}
T 2= & x s(x)-\int_{0}^{s(x)} \frac{s}{\left(1-\frac{1}{2} \psi\right)-\frac{1}{2} s} d s \\
= & x s(x)+2 s(x)+4\left(1-\frac{1}{2} \psi\right) \\
& \times \ln \left[1-\frac{s(x)}{2\left(1-\frac{1}{2} \psi\right)}\right]
\end{aligned}
$$

where

$$
s(x)=\frac{(2-\psi) x}{2+x}
$$

Dominance model

$$
\begin{gathered}
0 \leqq s, t \leqq x \\
T=x^{2}-x t(x)+\int_{0}^{t(x)} \frac{(2-\psi) t}{1-\psi t} d t \\
=x^{2}-x t(x)-\frac{2\left(1-\frac{1}{2} \psi\right) t(x)}{\psi} \\
-\frac{2\left(1-\frac{1}{2} \psi\right)}{\psi^{2}} \ln [1-\psi t(x)]
\end{gathered}
$$

where

$$
t(x)=\frac{x}{2-\psi(1-x)} .
$$

\section{Overdominance model}

$$
\begin{gathered}
0 \leqq s, t \leqq x \\
T=x^{2}-2\left[x t(x)-\int_{0}^{t(x)} \frac{(3-\psi) t-2 t^{2}}{1-\psi t} d t\right] \\
=x^{2}-2 x t(x)+\frac{2 t(x)^{2}}{\psi}+\frac{4-6 \psi-2 \psi^{2}}{\psi^{2}} \\
\quad \times\left[t(x)+\frac{1}{\psi} \ln (1-\psi t(x))\right]
\end{gathered}
$$

where

$$
t(x)=\frac{3-\psi(1-x)-\sqrt{[3-\psi(1-x)]^{2}-8 x}}{4} .
$$

Acknowledgements I thank Dr A. R. G. Owen for his help and advice with this problem, and an anonymous reviewer for suggesting the endpoint analysis. This paper was written while on a Postdoctoral Fellowship awarded by the Natural Sciences and Engineering Research Council of Canada.

\section{REFERENCES}

BEIG, D. 1972. The production of males in queenright colonies of Trigona (Scaptotrigona) postica. J. Apic. Res., 11, 33-39. BENNETT, J. H. 1957. Selectively balanced polymorphism at a sex-linked locus. Nature, 180, 1363-1364.

BENNETT, J. H. 1958. The existence and stability of selectively balanced polymorphism at a sex-linked locus. Aust. J. Biol. Sci., 11, 598-602.

BERKELHAMER, R. C. 1983. Intraspecific genetic variation and haplodiploidy, eusociality, and polygyny in the Hymenoptera. Evolution, 37, 540-545.

BOROFSKY, S. 1950. Elementary Theory of Equations. MacMillan, New York.

COLE, B. J. 1981. Dominance hierarchies in Leptothorax ants. Science, 212, 83-84. 
CONTEL, E. P. B. AND KERR, W. E. 1976. Origin of males in Melipona subnitida estimated from data of an isozymic polymorphic system. Genetica, 46, 271-277.

CROZIER, R. H. 1976. Counter-intuitive property of effective population size. Nature, 262, 384.

CROZIER, R. H. 1979. Genetics of sociality, pp. 223-286. In: Social Insects, Volume I. Edited by H. R. Hermann, Academic Press, New York.

CURTSINGER, J. W. 1980 . On the opportunity for polymorphism with sex-linkage or haplodiploidy. Genetics, 96, 995-1006.

EDWARDS, A. W. F. 1977. Foundations of Mathematical Genetics. Cambridge University Press. Cambridge.

FREE, J. B. 1955. The behaviour of egg-laying workers of bumble-bee colonies. Br. J. Anim. Behav., 3, 147-153.

HALDANE, J. B. S. 1926. A mathematical theory of natural and artificial selection. Part III. Proc. Camb. Phil. Soc., 23, 363-372.

HARTL, D. L. 1971. Some aspects of natural selection in arrhenotokous populations. Amer. Zoologist., 11, 309-325.

HONK, C. G. J. VAN, RÖSELER, P. F., VELTHUIS, H. M. W. AND HOOGEVEEN, J.C. 1981. Factors influencing the egg laying of workers in a captive Bombus terrestris colony. Behav. Ecol. Sociobiol., 9, 9-14.

LESTER, L. J. 1975. Population genetics of the Hymenoptera. Unpublished Ph.D. dissertation, University of Texas, Austin, U.S.A.

Lester, L. J. AND SElANDER, R. K. 1979. Population genetics of haplodiploid insects. Genetics, 92, 1329-1343.

MANDEL, S. P. H. 1959. Stable equilibrium at a sex-linked locus. Nature, 183, 1347-1348.

MAYO, O. 1976. Neutral alleles at $X$-linked loci. A cautionary note. Hum. Heredity, 26, 263-266.

METCALF, R. A., MARLIN, J. C. AND WHITT, G. S. 1975. Low levels of genetic heterozygosity in Hymenoptera. Nature, 257, 792-794.

OHTA, T. AND KIMURA, M. 1975. Theoretical analysis of electrophoretically detectable polymorphisms: models of very slightly deleterious mutations. Am. Naturalist, 109, 137 145.
OSTER, G. AND WILSON, E. O. 1978. Caste and Ecology in the Social Insects. Princeton University Press, Princeton, N.J., U.S.A.

OWEN, A. R. G. 1953. A genetical system admitting of two distinct stable equilibria under natural selection. Heredity, 7, 97-102.

OWEN, R. E. 1980. Population genetics of social Hymenoptera with worker produced males. Heredity, 45, 31-46.

OWEN, R. E. AND PLOWRIGHT, R. C. 1982. Worker-queen conflict and male parentage in bumble bees. Behav. Ecol. Sociobiol., 11, 91-99.

PAMILO, P. 1979. Genic variation at sex-linked loci: Quantification of regular selection models. Hereditas, 91, 129-133.

PAMILO, P. VARVIO-AHO, S. AND PEKKARINEN, A. 1978. Low enzyme gene variability in Hymenoptera as a consequence of haplodiploidy. Hereditas, 88, 93-99.

PAMILO, P. AND CROZIER, R. H. 1981. Genic variation in male haploids under deterministic selection. Genetics, 98, 199214.

PLATEAUX, L. 1981. The pallens morph of the ant Leptothorax nylanderi: Description, formal genetics, and study of populations. pp. 63-74. In: Biosystematics of Social Insects. Edited by P. E. Howse and J. L. Clement. Academic Press, New York.

POMEROY, N. 1979. Brood bionomics of Bombus ruderatus in New Zealand (Hymenoptera: Apidae). Can. Entomol., 112, 865-874.

RICHARIS, O. W. 1946. Observations on Bombus agrorum (Fabricius) (Hymen., Bombidae). Proc. R. Entomol. Soc. (Lond)., 21, 66-71.

SNYDER, T. P. 1974. Lack of allozymic variability in three bee species. Evolution, 28, 687-689.

SPRADBERY, J. P. 1973. Wasps: An Account of the Biology and Natural History of Solitary and Social Wasps. University of Washington Press, Seattle, U.S.A.

WILSON, E. O. 1963. Social modifications related to rareness in ant species. Evolution, 17, 249-253. 